\title{
Fully Differential Single-Photon Double Ionization of Neon and Argon
}

\author{
F. L. Yip, ${ }^{1, *}$ T. N. Rescigno, ${ }^{2}$ C. W. McCurdy, ${ }^{2,3}$ and F. Martín ${ }^{1,4, \dagger}$ \\ ${ }^{1}$ Departamento de Química, Modulo 13, Universidad Autónoma de Madrid, 28049 Madrid, Spain \\ ${ }^{2}$ Lawrence Berkeley National Laboratory, Chemical Sciences, and Ultrafast X-ray Science Laboratory, Berkeley, \\ California 94720, USA \\ ${ }^{3}$ Department of Chemistry, University of California, Davis, California 95616, USA \\ ${ }^{4}$ Instituto Madrileño de Estudios Avanzados en Nanociencia, Cantoblanco, 28049 Madrid, Spain \\ (Received 3 September 2012; revised manuscript received 15 March 2013; published 22 April 2013)
}

\begin{abstract}
Triply differential cross sections are calculated for one-photon double ionization of neon and argon at various photon energies and electron energy sharings by using a frozen-core treatment to represent the remaining electrons of the residual ion. Angular distributions agree well with all existing experimental data, showing that in spite of its simplicity the method can treat the double ionization of complex targets reliably. A comparison of the cross sections for helium, neon, and argon into the same final state symmetry at the same relative excess energies reveals a distinctive signature of the role of electron correlation in each target.
\end{abstract}

DOI: 10.1103/PhysRevLett.110.173001

PACS numbers: $32.80 . \mathrm{Fb}, 34.10 .+\mathrm{x}$

One-photon double photoionization (DPI) of atomic targets is a fundamental problem that has been the subject of significant investigation because of the explicitly correlated nature of the process. The simplest target where DPI can occur is helium, for which experiments using coincidence measurements of the resulting Coulomb fragments allow description of the fully differential electron dynamics. In parallel, $a b$ initio theoretical treatments of helium DPI have produced results in excellent agreement with experiment: Double ionization of helium can be considered a solved problem (see, e.g., Refs. [1-5], and references therein). In contrast, for heavier rare-gas atoms, the volume of experimental measurements of one-photon DPI greatly outweighs the theoretical data, which have been confined to model treatments [6] and parameterization of the process $[7,8]$. At the same time, significant interest in studying rare-gas targets in laser-atom interactions has evolved beyond the single-photon perturbative limit of DPI. Processes such as high-harmonic generation [9], attosecond streaking [10], and multiphoton ionization [11] with coherent light sources represent some of the most recent examples of the evolving scope for investigating multielectron dynamics in rare gases. Application of precise and kinematically complete experimental techniques has outpaced the capacity for absolute theoretical treatments to fully represent the total correlated dynamics in rare-gas targets beyond helium. For single-photon DPI of neon or argon, no accurate fully $a b$ initio description of this process has been achieved.

Here we report the results of a method that makes use of a fully correlated grid-based expansion to describe the electrons ejected into the double continuum in the presence of the remaining bound electrons that advances our theoretical capacity to describe DPI in many-electron atomic targets. The key to this framework is the construction of an atomic orbital basis that represents the bound $N-2$ electrons, which will remain frozen throughout the double ionization process [12]. Thus the final state represents two indistinguishable continuum electrons that will share the excess photon energy in the presence of those electrons remaining bound to the parent ion. The proper description of the angular distributions and energy sharing inherently requires an accurate treatment of electron correlation.

In this Letter, we will show that, in spite of the apparent simplicity of the method, the accuracy of the calculated fully differential cross sections for DPI of many-electron rare-gas atoms is nearly comparable to that currently achieved for helium. This is most remarkable, since the two active electrons arise from the $p$ shell, which contains six equivalent electrons. The accuracy is illustrated by the excellent agreement between the present and experimental results for $\mathrm{Ne}$ and Ar targets at various photon energies and both equal and unequal energy sharing. The method is used to gauge the role of electron correlations in the DPI of these complex targets, and, in particular, we show that interpretations based on previous knowledge of helium DPI must be taken with great caution.

The complexity of the DPI process for neon or argon relative to helium is evident from the existence of three possible final states of the double cation when ionizing out of the $n p^{6}$ valence subshell. The overall symmetry of the target initial state is ${ }^{1} S^{e}$, while that of the residual cation in the $n p^{4}$ configuration is ${ }^{1} S^{e},{ }^{1} D^{e}$, or ${ }^{3} P^{e}$. To preserve the initial ${ }^{1} S^{e}$ symmetry, the fully active electrons must bear the same symmetry as the cation; i.e., the coupling must be such that the two-active-electron part of the initial state has ${ }^{1} S^{e},{ }^{1} D^{e}$, or ${ }^{3} P^{e}$ symmetry. Fine structure details have not been included.

With the symmetry of the two active and $N-2$ frozen electrons determined, we must consider the nature of 
interactions between them. As a first approximation to ignore the complications due to the open-shell nature of the residual states, we represent the Hamiltonian of the photoionized electrons in a full two-electron picture (atomic units are used throughout)

$$
H=h(1)+h(2)+\frac{1}{r_{12}},
$$

where $1 / r_{12}$ is the Coulomb repulsion potential. This Hamiltonian represents the interaction of the active electrons with each other in the field of the remaining $N-2$ core and valence electrons. Each outgoing electron sees an effective one-body Hamiltonian $h$ of the form

$$
h=-\frac{1}{2} \nabla^{2}-\frac{Z}{r}+\sum_{o}\left(2 J_{o}-K_{o}\right)+4 J_{n p}^{0},
$$

where the first two terms in Eq. (2) are the one-electron kinetic energy operator and nuclear attraction potential, respectively, $J$ represents a Coulomb operator, $K$ is an exchange operator, and the sum runs over occupied closed-shell orbitals. This sum accurately represents the direct and exchange potentials induced by the close-shell electrons on the outgoing electrons. The last term reduces the open-shell potential felt by the $n p$ valence electrons to a pure Coulomb monopole interaction with four electrons held fixed in $n p$ orbitals of the neutral atom and is, in fact, the effective potential obtained by averaging the Coulomb interactions with any one of the ${ }^{1} S^{e},{ }^{3} P^{e}$, and ${ }^{1} D^{e}$ states of residual $n p^{4}$ dication over $M_{L}$ projections. Since absorption of a single photon populates continuum states with an overall ${ }^{1} P^{o}$ symmetry, the dipole approximation implies that the symmetry of the fully active electrons in the final state is ${ }^{1} P^{o}$ (for the $n p^{4}{ }^{1} S^{e}$ state of the dication), ${ }^{3} P^{o}$ and ${ }^{3} D^{o}$ (for the $n p^{4}{ }^{3} P^{e}$ state of the dication), and finally ${ }^{1} P^{o}$, ${ }^{1} D^{o}$, and ${ }^{1} F^{o}$ (for the $n p^{4}{ }^{1} D^{e}$ state of the dication).

The orbitals representing those electrons that remain frozen are chosen to be Hartree-Fock orbitals. They are expanded in a finite-element discrete variable representation (FEM-DVR) basis [13] defined in two distinct regions: an inner region consisting of a few finite elements and an outer region spanned by pure primitive FEM-DVR functions. It is over the inner orbital region that the first few FEM-DVR basis functions are transformed into an orthonormal basis of atomic orbitals by taking linear combinations of the primitive DVR functions. The part of the initial states corresponding to the fully active electrons is expanded in a product basis formed from FEM-DVR functions and coupled spherical harmonics (see Refs. [12,14] for details).

The present formalism ignores exchange between the ionized electrons and the residual $2 p^{4}$ core. The initial states for the two active electrons corresponding to the three possible residual dication states were computed as the lowest eigenstates of Eq. (1) with the appropriate spin and spatial symmetries mentioned above (see Supplemental Material [15]). The space of the two active electrons can thus not be held orthogonal to the $2 p$ orbitals of the core, and therefore the effective Hamiltonian would contain energy-dependent operators if exchange with the core were included completely [16]. That would invalidate the method for extracting the double ionization amplitude described below. To gauge the importance of the neglect of exchange, we added to the Hamiltonian of Eq. (2) a spherically averaged exchange potential obtained from accurate multiconfiguration self-consistent field calculations. The fully differential cross sections obtained by using this extended Hamiltonian are very close to those reported below [17]. Therefore the dominant interactions are reasonably well represented by the present approach in which all but two electrons are held fixed in valence orbitals of the neutral.

Using these initial states with two fully correlated electrons in the presence of a frozen target ion, we can extract the double ionization amplitudes which contain the fully correlated dynamics of the outgoing electrons for each residual ionic channel. This involves solving, for each of the $L S$ couplings allowed by the dipole selection rule (see above), a first-order driven Schrödinger equation within the dipole approximation:

$$
\left(E_{0}+\hbar \omega-H\right)\left|\Psi_{\mathrm{sc}}^{+}\right\rangle=(\epsilon \cdot \mu)\left|\Phi_{0}\right\rangle,
$$

where $\left|\Psi_{\mathrm{sc}}^{+}\right\rangle$is an outgoing wave function with the appropriate final state coupling. The outgoing wave boundary conditions are imposed on the solution to Eq. (3), $\Psi_{\mathrm{sc}}^{+}$, by using exterior complex scaling [18]. The amplitudes for double ionization that lead directly to the triply differential cross sections (TDCS) are extracted by using a surface integral formalism computed from the partial wave components of the scattered wave along with appropriate testing functions $[12,18]$. In the present case, these testing functions are obtained as continuum states of the onebody Hamiltonian $h$ in Eq. (2).

The various TDCS calculated by applying this theoretical framework to DPI of neon leaving the residual dication in each of the final state couplings are shown in Fig. 1. The angular distributions calculated for equal energy sharing are presented for various experimental conditions along with the only existing measurements by Krässig et al. [19] in the first five panels. The results were obtained in the velocity gauge; however, they are practically indistinguishable from those obtained in the length gauge (see Supplemental Material [15]). Notice that for those panels on the left where the Stokes parameter of the photon differs significantly from unity, calculation of the TDCS requires a weighted combination of the $x$ - and $z$-polarization cross sections. In addition, in order to calculate ${ }^{3} P^{e}$ and ${ }^{1} D^{e}$ TDCS, a sum over final states of the possible $M_{L}$ values averaged over the degenerate possible initial orientations is required.

The absolute TDCS results have been normalized to the maximum of the independent experimental points in each panel. The agreement between these results for each final 

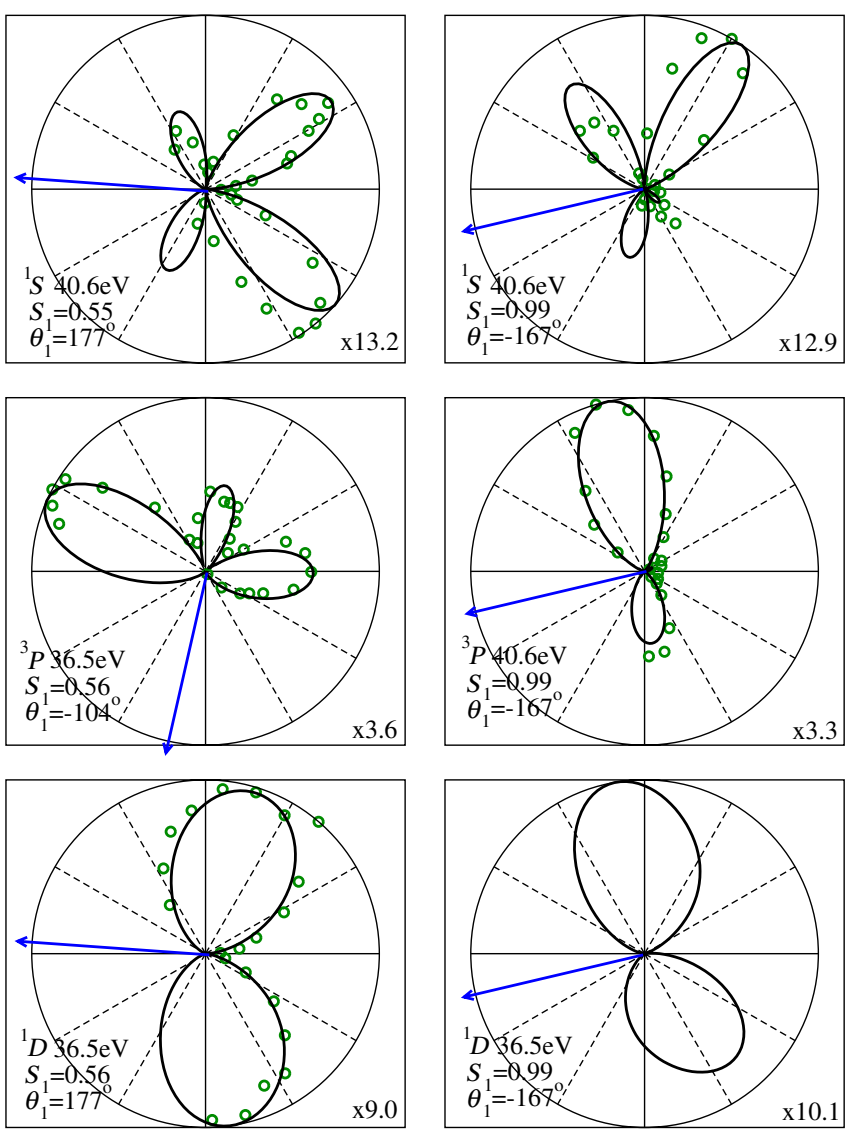

FIG. 1 (color online). TDCS results for DPI from neon (black curve) with equal energy ionized electrons under various experimental conditions. Experimental points (green circles) in the first five panels are those of Krässig et al. [19]. The final state symmetry of the residual dication and photon energy are described in each panel. The fixed electron indicated by the blue arrow is measured at different angles $\theta_{1}$ in the plane perpendicular to the photon beam, as indicated. $S_{1}$ is the Stokes parameter that describes the relative excess of linear polarization along the major and minor axis of the polarization ellipse. $S_{1}=1$ corresponds to perfectly linearly polarized light along the horizontal $z$ axis. Factors at the bottom right of each panel denote the magnitudes of the calculated TDCS in $\mathrm{b} / \mathrm{eV} / \mathrm{sr}^{2}$.

state symmetry is excellent, particularly for the cases of ${ }^{3} P^{e}$ and ${ }^{1} D^{e}$ residual ion states. Comparing the present angular distributions with the equal energy sharing results calculated by Malcherek, Maulbetsch, and Briggs [6], who used a model final state wave function, reveals generally good agreement, although we find better agreement with the experimental results in the case of the ${ }^{3} P^{e}$ cationic state, where the triplet nature of the outgoing electrons is distinguished from the other two symmetries. Each possible final state, however, possesses its own symmetry considerations at equal energy sharing, which imprints the observed angular distributions and significantly differentiates them from one another.

We turn now to the argon case. To further examine the DPI results calculated for a particular final state symmetry
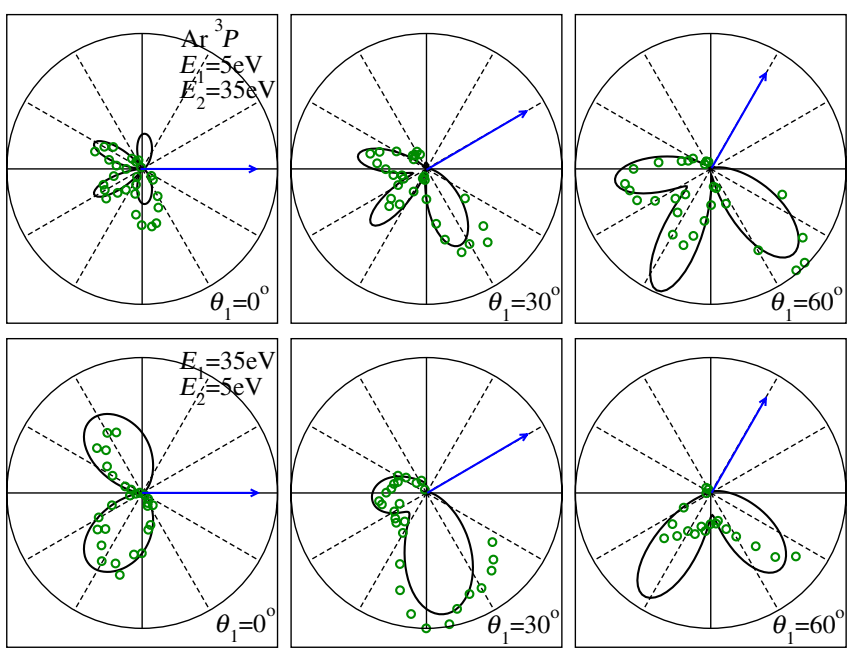

FIG. 2 (color online). Internormalized TDCS results calculated for argon (black curve) leaving the dication in a $3 p^{4}{ }^{3} P^{e}$ final state with excess photon energy $40 \mathrm{eV}$ above the DPI threshold. Experimental points (green circles) are those measured by Bolognesi et al. [20]. The fixed electron carries kinetic energy $E_{1}$ and is indicated by the blue arrow measured at angle $\theta_{1}$ in the plane perpendicular to the photon beam containing the polarization, which lies along the horizontal axis in all panels. The radial length in each panel is $2.2 \mathrm{~b} / \mathrm{eV} / \mathrm{sr}^{2}$.

of the residual ion, we focus on comparisons of the angular distributions given for the ${ }^{3} P^{e}$ symmetry at unequal energy sharing, for which, to our knowledge, no nonparameterized theoretical results have been reported. Experimental measurements by Bolognesi et al. [20] for DPI leaving the ${ }^{3} P^{e}$ ionic state of argon are compared in Fig. 2 with the present results obtained in the velocity gauge for pure linearly polarized light leaving the ejected electrons in the double continuum with $40 \mathrm{eV}$ excess energy to share. Gauge invariance is not as good as in the Ne case, due possibly to a poorer description obtained in the length gauge (see Supplemental Material [15]). Since the experimental results are internormalized, the absolute TDCS results have been referenced to the experimental hit count in the upper right panel, thereby permitting a direct comparison of the calculated TDCS with experiment. As can be seen, the major features of the angular distributions are well represented by the present calculation. We also find superb agreement in the variation of the magnitudes of the cross sections. This agreement between the present and experimental results for the unequal energy sharing case, which is less dominated by symmetry selection rules than when $E_{1}=E_{2}$ and, consequently, more difficult to describe, is noteworthy and provides some evidence that a significantly faithful representation of the electron dynamics is contained even within the limitations of the approximate Hamiltonian for each of the two outgoing electrons contained in Eq. (2). Nevertheless, some quantitative discrepancies with experiment remain, especially in the lower right panel of Fig. 2. These may be due to the poorer gauge 


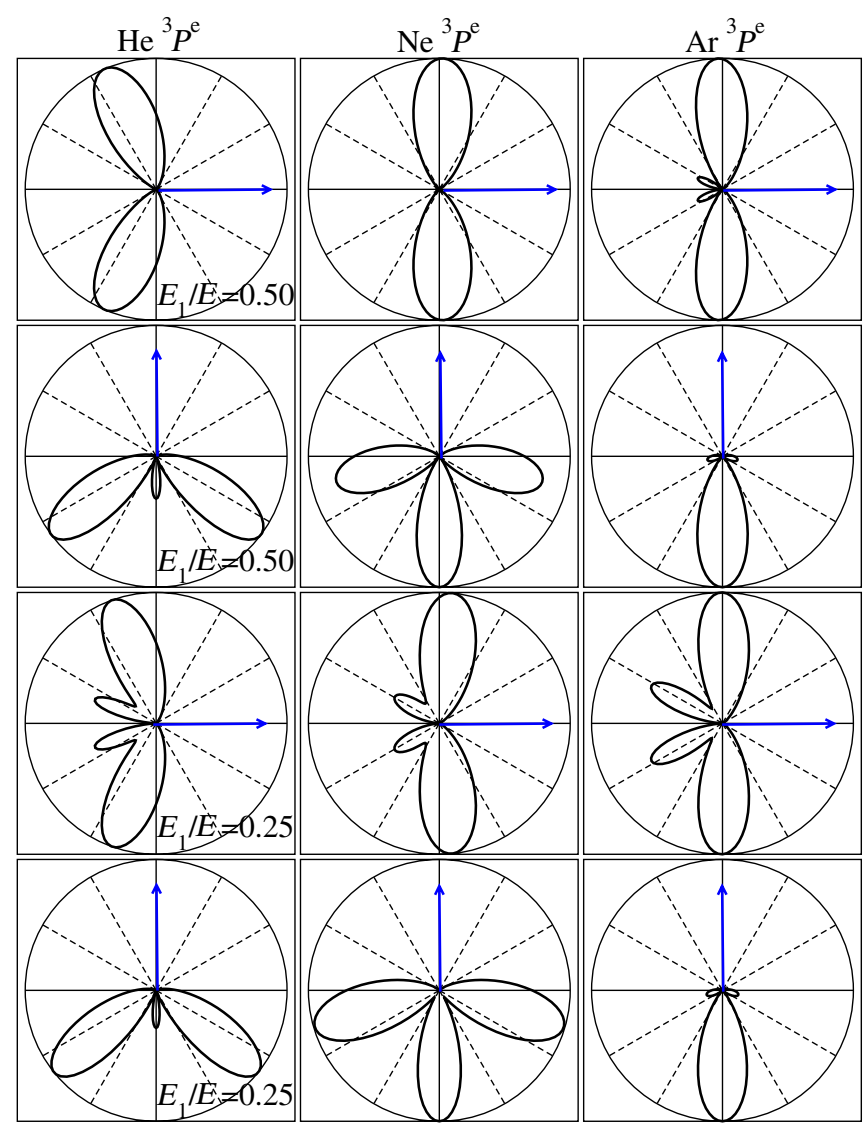

FIG. 3 (color online). TDCS results (black curves) for DPI from doubly excited $2 p^{2}{ }^{3} P^{e}$ helium (first column), neon leaving the ion in the $2 p^{4}{ }^{3} P^{e}$ configuration (second column), and argon leaving the residual $3 p^{4}{ }^{3} P^{e}$ dication (third column). The photon energy is 1.303 times the double IP. The blue arrow denotes the fixed electron direction relative to the polarization direction (horizontal axis) carrying the indicated energy sharing. Cross sections are normalized to unity.

invariance of the Ar calculations compared to the $\mathrm{Ne}$ ones or to the fact that, for unequal energy sharing, the absence of symmetry selection rules makes the physics more challenging.

The previous examples demonstrate the distinct and varied character of the angular distribution for DPI from multielectron $n p^{6}$ rare-gas targets into a particular $L S$ channel. It is not surprising that results for neon and argon at lower photon energies differ from those of ground state helium, since valence DPI for the first open channel proceeds out of distinct angular momentum subshells and, consequently, leads to nonequivalent final state couplings of the residual target. Therefore, a better comparison is afforded by considering DPI from corresponding initial valence subshell occupations. In Fig. 3, the unitnormalized angular distributions for DPI from doubly excited $2 p^{2}{ }^{3} P^{e}$ helium are compared with neon and argon leaving the residual dication in the most-stable $2 p^{4}{ }^{3} P^{e}$ and $3 p^{4}{ }^{3} P^{e}$ configurations, respectively. The $2 p^{2}{ }^{3} P^{e}$ doubly excited state of helium is prepared by projecting the $1 s$ and $2 s$ orbitals of $\mathrm{He}^{+}$from the expansion of the wave function. This is equivalent to a configuration interaction calculation in which all configurations containing electrons in the $1 s$ and $2 s$ orbitals of $\mathrm{He}^{+}$are excluded. Results are shown for DPI with photon energy 1.303 times the corresponding double IP of the target with the energy shared both equally (upper panels) and asymmetrically (lower panels), focusing on parallel and perpendicular geometries of the fixed electron. At these relatively modest photon energies, the resulting angular distributions are greatly influenced by the character of their initial state environments. Considering the common symmetry imposed by the ${ }^{3} P^{e}$ target coupling of the outgoing electrons, the significant differences in the cross sections of the heavier atoms compared to helium highlights the role of the $p$ core electrons in determining how the two escaping electrons correlate. Furthermore, the TDCS results of neon and argon also display visible differences when compared to each other, despite the similar treatment of the outgoing and frozen electrons. For both equal and asymmetric energy sharing, both geometries reflect a significant variation of angular correlation when going from excited helium to neon and even more when going from $\mathrm{Ne}$ to Ar, especially for perpendicular ejection. In the parallel geometry, secondary lobes appear as one moves to heavier rare gases. These lobes lie almost back to back with respect to the direction of ejection of the first electron, indicating a variation in angular correlation resulting from the slightly larger radial extent and additional nodal structure of the $n p$ orbitals of argon compared to neon. In the perpendicular case, the TDCS evolves from a nearly two-lobed shape for $\mathrm{He}$ to a three-lobed one for $\mathrm{Ne}$ and to a nearly back-to-back one-lobed shape for Ar. Again, this result indicates a significant change of angular correlation in Ar. Interestingly, this comparison in the perpendicular case does not seem to depend on the energy sharing between electrons.

In conclusion, theoretical calculations of the fully differential DPI cross sections for one-photon DPI of Ne and $\mathrm{Ar}$, in which the residual electrons are represented by a frozen core, give results in good agreement with existing experimental data. A comparison of the calculated cross sections for helium, neon, and argon into the same final state symmetry at the same relative excess energies reveals the signatures of the role of electron correlation. The differences between targets are due to the different spatial extent and nodal structures of the $n p$ orbitals from which the electrons are ejected. We therefore conclude that, for this particular process, angular correlation plays a very different role in Ar than in Ne. Finally, the good agreement with experiment suggests that these methods can be used to accurately study other correlated multielectron dynamics in rare-gas targets with more complexity than helium. The latter is particularly important for few-photon ionization processes involving attosecond pulses, whose short 
duration would allow one to follow the dynamics of electron correlation in real time.

This work was accomplished with an allocation of computer time from Mare Nostrum BSC and NERSC and partially supported by the MICINN Projects No. FIS201015127, No. ACI2008-0777, and No. CSD 2007-00010, the ERA-Chemistry Project No. PIM2010EEC-00751, the European grant MC-ITN CORINF, the European COST Action CM0702, and the Advanced Grant of the European Research Council No. XCHEM 290853. C.W.M. and T.N.R. acknowledge support from U.S. Department of Energy Office of Basic Energy Sciences, Division of Chemical Sciences Contract No. DE-AC02-05CH11231. F.L. Y. acknowledges a postdoc contract from MICINN.

*Present address: Department of Science and Mathematics, California Maritime Academy, Vallejo, CA 94590, USA. †fernando.martin@uam.es

[1] R. Wehlitz, F. Heiser, O. Hemmers, B. Langer, A. Menzel, and U. Becker, Phys. Rev. Lett. 67, 3764 (1991).

[2] V. Mergel et al., Phys. Rev. Lett. 80, 5301 (1998).

[3] K. Soejima, A. Danjo, K. Okuno, and A. Yagishita, Phys. Rev. Lett. 83, 1546 (1999).

[4] A. Huetz and J. Mazeau, Phys. Rev. Lett. 85, 530 (2000).

[5] L. Avaldi and A. Huetz, J. Phys. B 38, S861 (2005).

[6] A. W. Malcherek, F. Maulbetsch, and J. S. Briggs, J. Phys. B 29, 4127 (1996).
[7] L. Malegat, P. Selles, P. Lablanquie, J. Mazeau, and A. Huetz, J. Phys. B 30, 263 (1997).

[8] L. Argenti and R. Colle, J. Phys. B 41, 245205 (2008).

[9] P. M. Paul, E. S. Toma, P. Breger, G. Mullot, F. Aug, P. Balcou, H. G. Muller, and P. Agostini, Science 292, 1689 (2001).

[10] M. Schultze et al., Science 328, 1658 (2010).

[11] B. Bergues et al., Nat. Commun. 3, 813 (2012).

[12] F. L. Yip, C. W. McCurdy, and T. N. Rescigno, Phys. Rev. A 81, 053407 (2010).

[13] T. N. Rescigno and C.W. McCurdy, Phys. Rev. A 62, 032706 (2000).

[14] F. L. Yip, F. Martín, C. W. McCurdy, and T. N. Rescigno, Phys. Rev. A 84, 053417 (2011).

[15] See Supplemental Material at http://link.aps.org/ supplemental/10.1103/PhysRevLett.110.173001 for the gauge dependence of the results, discussion of the energies of the bound states, and effect of the neglected exchange interactions.

[16] M.E. Riley and D. G. Truhlar, J. Chem. Phys. 65, 792 (1976).

[17] F. L. Yip, T. N. Rescigno, C. W. McCurdy, and F. Martín (to be published).

[18] C. W. McCurdy, M. Baertschy, and T.N. Rescigno, J. Phys. B 37, R137 (2004).

[19] B. Krässig, S. J. Schaphorst, O. Schwarzkopf, N. Scherer, and V. Schmidt, J. Phys. B 29, 4255 (1996).

[20] P. Bolognesi, M. Zitnik, L. Malegat, P. Selles, G. Turri, M. Coreno, R. Camilloni, and L. Avaldi, J. Phys. B 37, 2285 (2004). 